\title{
Malignant phyllodes tumor of the prostate and seminal vesicle: an unusual presentation
}

\author{
Roman Leonid Kleynberg, MD, ${ }^{1}$ Yasmeen Kabir, MD, ${ }^{1}$ \\ Punprapai Boriboonsomsin, MD, ${ }^{2}$ and Jeffrey M. Miller, $\mathrm{MD}^{2}$ \\ ${ }^{1}$ Department of Internal Medicine and ${ }^{2}$ Division of Hematology/Oncology, David Geffen School of Medicine at UCLA, Olive \\ View - UCLA Medical Center, Sylmar, California
}

\begin{abstract}
58-year-old man was admitted to hospital with gross hematuria and a history of 1 a prostate nodule that had been unchanged in size over a period of 3 years. A digital rectal examination revealed a nodule, confirmed by pelvic computed tomography and magnetic resonance imaging scans. Microscopic findings from a transrectal ultrasound-guided biopsy of the prostate revealed phyllodes tumor of the prostate and seminal vesicle, with well-differentiated fibrosarcoma and undifferentiated sarcoma. An initial prostate-specific antigen (PSA) value was 2 $\mathrm{ng} / \mathrm{mL}$ (normal, $<4 \mathrm{ng} / \mathrm{dL}$ ). Over time, the mass grew in size and caused abdominal bloating, bladder outlet obstruction, and kidney failure. A radical prostatectomy was performed and the patient had an uneventful postoperative course. Thereafter, the patient received adjuvant radiation therapy. A few months after surgery, the symptoms worsened and imaging revealed a recurrence and metastasis to his lungs. The patient is currently receiving palliative chemotherapy.
\end{abstract}

\section{Discussion}

We discuss here the diagnosis, classification, histology, treatment, and prognosis of this rare tumor. Phyllodes tumor of the prostate and/or seminal vesicle is very rare. Histologically, phyllodes tumor of the prostate, one of the epithelialstromal tumors, resembles its counterpart in the female breast tissue. The median age of presenta-

\footnotetext{
Manuscript received June 25, 2012; accepted October 23, 2012. Correspondence Roman Leonid Kleynberg, MD, David Geffen School of Medicine at UCLA, Olive View - UCLA Medical Center, Department of Internal Medicine, 14445 Olive View Drive, Sylmar CA 91342 (roman.kleynberg@ ucla.edu).

Disclosures None of the authors has any disclosures.
}

tion of patients with these tumors is about 55 years, (range, 22-86 years). ${ }^{1}$ Patients often present with obstructive symptoms such as increased urinary hesitancy, terminal dribbling, and incomplete voiding. Others present with hematuria.

Phyllodes tumor is usually diagnosed after surgical removal of the prostate gland and not by needle biopsies because it is difficult to study the architecture of biopsy samples. Phyllodes tumor of the prostate in previous reported cases has been pathologically classified as either low-grade (benign), intermediate, or high-grade (malignant) based on the stromal-epithelial ratio, stromal cellularity, cytological atypia, necrosis, and mitotic activity. ${ }^{1,2}$ Microscopically, it has a distinct pattern of epithelial-lined cystic spaces, with epithelial-lined leaf-like stromal projections. The presence of both epithelial and stromal elements is necessary to make the diagnosis.

The definitive treatment of phyllodes tumor of the prostate is radical surgery. It is important to obtain negative margins to decrease the chance of recurrence. In addition, because of the rarity of phyllodes tumor, the treatment of metastatic and recurrent cases has not been well established. In previous case reports, chemotherapy with ifosfamide and doxorubicin for lung metastasis after radical prostatectomy has been shown to be effective in addition to adjuvant irradiation. ${ }^{3}$ Radiation has been also studied in those patients with metastatic tumors, ${ }^{4}$ and the efficacy of VIP (etoposide, ifosfamide, and cisplatin) chemotherapy as induction therapy has also been reported. ${ }^{5}$ Phyllodes tumor recurrence after radical resection is common, occurring in about $65 \%$ of patients. Recur(c) 2013 Frontline Medical Communications www.CommunityOncology.net 
rence rates range between $50 \%$ for low-grade tumors and $100 \%$ for high-grade tumors. ${ }^{6}$

Vimentin and desmin immunoreactivity in phyllodes tumor is not specific, but it is immunoreactive in prostatic stromal hyperplasia with atypia. ${ }^{1} \mathrm{CD} 34$ is also nonspecific in phyllodes tumor and stromal tumors of uncertain malignant potential. Phyllodes tumor is a unique entity that can be differentiated from gastrointestinal stromal tumors by the absence of c-KIT and CD34 expression. Previous case reports have shown that PSA values in these types of patients are within normal limits or slightly elevated, ${ }^{5}$ however PSA values can also be elevated. Increasing serum PSA levels are observed in benign prostatic hyperplasia, prostatitis, and prostate cancer.

\section{Conclusion}

Phyllodes tumor of the prostate and seminal vesicle is a challenging disease to diagnosis and treat. Mortality is high even in patients that are treated without metastasis and recurrence is common. Thus, early recognition and treatment of this disorder is essential to reduce the associated morbidity and mortality.

\section{References}

1. Bostwick DG, Hossain D, Qian J, et al. Phyllodes tumor of the prostate: Long-term follow-up study of 23 cases. J Urol. 2004;172: 894-899.

2. Young JF, Jensen PE, Wiley CA. Malignant phyllodes tumor of the prostate. A case report with immunohistochemical and ultrastructural studies. Arch Pathol Lab Med. 1992;116:296-299.

3. Lam KC, Yeo W. Chemotherapy induced complete remission in malignant phyllodes tumor of the prostate metastasizing to the lung. J Urol. 2002;168:1104-1105.

4. Yamamoto S, Ito T, Miki M, Serizawa H, Maekawa S, Furusato M. Malignant phyllodes tumor of the prostate. Int J Urol. 2000;7:378381.

5. Sakura M, Tsukamoto T, Yonese J, Ishikawa Y, Aoki N, Fukui I. Successful therapy of a malignant phyllodes tumor of the prostate after postoperative local failure. Urology. 2006;67:845.e11-e13.

6. Aguilar B, Villalobos M, Sotomayor M. Phyllodes tumor of the prostate. Urology. 2009;73:272. 